\title{
Hot off the breath: a big step forward for idiopathic pulmonary fibrosis
}

\author{
Christopher J Ryerson, ${ }^{1}$ Harold R Collard ${ }^{2}$
}

Idiopathic pulmonary fibrosis (IPF) is a chronic progressive interstitial lung disease of unknown etiology ${ }^{1}$ with a median survival of 3 years from the time of diagnosis. ${ }^{2}$ The recent decade has seen a tremendous investment in IPF clinical research, and as a result, we have moved beyond traditional treatment approaches (prednisone and other immunomodulatory therapies) toward clinical trials of potential antifibrotic therapies. ${ }^{3}$ As recently as 2011, however, an international guideline committee concluded that there remained insufficient evidence to support the regular use of any IPF pharmacotherapy. ${ }^{1}$ Because of this, there has been no consensus standard of care for IPF patients.

This will likely change with the recent publication of three landmark studies. ${ }^{4-6}$ Two novel therapies, pirfenidone and nintedanib, were found to slow disease progression in patients with IPF, while a third therapy, acetylcysteine, was found to have no effect. We believe these studies will transform IPF management and lead to the establishment of a universal standard of care. We discuss each of the three trials below, followed by a discussion of their clinical and clinical research implications.

\section{ASCEND (PIRFENIDONE)}

Pirfenidone is an orally available antifibrotic agent with multiple potential mechanisms of action. Early clinical trials suggested that pirfenidone could slow lung function decline in patients with $\mathrm{IPF}^{7-9}$ prompting two parallel, randomised, double-blind, placebo-controlled, phase III clinical trials (the CAPACITY studies). ${ }^{10}$ There was a significant benefit in the primary endpoint of change in FVC over 72 weeks in one of the CAPACITY studies but not in the other. Pirfenidone was associated with an increased risk of gastrointestinal (eg, nausea and stomach

\footnotetext{
${ }^{1}$ Department of Medicine, University of British Columbia, Vancouver, British Columbia, Canada; ${ }^{2}$ Department of Medicine, University of California, San Francisco, San Francisco, California, USA

Correspondence to Dr Christopher J Ryerson, Department of Medicine \& Centre for Heart Lung Innovation, University of British Columbia, St. Paul's Hospital, Ward 8B, 1081 Burrard Street, Vancouver, British Columbia, Canada V6Z 1Y6; chris.ryerson@hli.ubc.ca.
}

discomfort) and dermatological (eg, photosensitivity) adverse effects, however, these were generally manageable, and most patients were able to continue at the full dose for the duration of the study. Based largely on the CAPACITY results, pirfenidone was approved for the treatment of IPF in several countries.

An additional phase III trial was required by the US Food and Drug Administration (FDA) to reconcile the discordant primary endpoint results from the two CAPACITY trials. This trial, the ASCEND study, was a randomised controlled trial that included 555 IPF patients with mild to moderate physiological impairment. ${ }^{5}$ Compared to placebo, pirfenidone $(2403 \mathrm{mg}$ daily taken in three divided doses) resulted in a relative reduction of $47.9 \%$ in the proportion of patients with a 10\% absolute FVC decline, or death over 52 weeks, as well as improvements in progression-free survival and 6 min walk distance. A prespecified analysis of the 1247 patients that were randomised to placebo, or full dose of pirfenidone in the combined CAPACITY and ASCEND trials suggested a reduction in 52-week mortality (HR 0.52, 95\% CI 0.31 to $0.87, p=0.01)$. The side effect profile in ASCEND appeared similar to CAPACITY.

There were some differences in the ASCEND and CAPACITY study populations. Patients in ASCEND had worse overall baseline pulmonary function, were more likely to be using supplemental oxygen, and were less likely to have concurrent airflow limitation compared to CAPACITY patients. In addition, all patients in ASCEND underwent a central review of radiological and pathological findings prior to enrolment, resulting in a highly scrutinised and potentially more refined population. Finally, patients in ASCEND were treated for 52 as opposed to 72 weeks.

\section{INPULSIS (NINTEDANIB)}

Nintedanib is an orally available tyrosine kinase inhibitor that is active against a large number of tyrosine kinases, including vascular endothelial growth factor (VEGF), fibroblast growth factor (FGF), and platelet-derived growth factor (PDGF). The TOMORROW study was a phase II trial that compared placebo to four doses of nintedanib, and it suggested that nintedanib $150 \mathrm{mg}$ twice daily slowed the rate of decline in FVC and reduced the risk of acute exacerbation. ${ }^{11}$

The results of the TOMORROW study prompted two parallel, randomised, double-blind, placebo-controlled, phase III clinical trials (the INPULSIS trials). ${ }^{4}$ These identical studies compared nintedanib (150 mg twice daily) to placebo in 1066 patients with mild to moderate physiological impairment. Both trials demonstrated a reduction in the rate of decline in FVC over 52 weeks (relative reduction of $52 \%$ and $45 \%$ ) over the 52 -week study period. The key secondary endpoints, time to first IPF exacerbation and quality of life (St George's Respiratory Questionnaire), were improved in one trial but not the other. There was no significant effect of nintedanib on time to death in a prespecified pooled analysis of both trials (HR 0.70; 95\% CI 0.43 to $1.12, \mathrm{p}=0.14)$. Nintedanib was associated with diarrhoea $(62 \%$ in nintedanib vs $18 \%$ in placebo), but most patients tolerated the full dose of nintedanib for the majority of the study.

\section{PANTHER-IPF (ACETYLCYSTEINE)}

Acetylcysteine is a precursor to the antioxidant, glutathione, that had been used as a treatment for IPF (either alone or in combination) based largely on data from a randomised double-blind trial (the IFIGENIA study) that compared acetylcysteine to placebo in patients who were all treated with prednisone and azathioprine. ${ }^{12}$ The IFIGENIA study demonstrated that patients receiving acetylcysteine had less of a decline in lung function, however, the absence of an acetylcysteine monotherapy arm, and a true no-therapy arm limited any direct conclusions about its efficacy.

This limitation led to the design of a second phase III clinical trial of acetylcysteine in IPF (the PANTHER-IPF trial). The objective of the PANTHER-IPF study was to determine whether acetylcysteine or the combination of acetylcysteine with prednisone and azathioprine reduced the rate of decline in FVC over 60 weeks in IPF patients with mild to moderate physiological impairment. The study was interrupted by the data safety monitoring board due to an excess number of deaths and hospitalisations in the acetylcysteine with prednisone and azathioprine arm. ${ }^{13}$ This triple therapy arm was discontinued, but the acetylcysteine monotherapy and placebo arms of this study remained blinded and were allowed to continue. 
The results from these two remaining PANTHER-IPF arms have now been published. ${ }^{6}$

The second PANTHER-IPF report included 264 IPF patients who had been treated with either acetylcysteine monotherapy or placebo for 60 weeks. ${ }^{6}$ There was no impact of acetylcysteine on the primary endpoint of change in FVC and most secondary endpoints. Patients on acetylcysteine had fewer gastrointestinal adverse effects, but an increase in nonfatal cardiac events. There was no significant between-group differences with respect to the primary endpoint for any of the subgroup analyses.

\section{CLINICAL AND CLINICAL RESEARCH IMPLICATIONS}

Pirfenidone and nintedanib both appear to slow the rate of disease progression in IPF with manageable adverse effects, and will likely become standard of care worldwide in the next year. Conversely, acetylcysteine does not appear to have a role in the treatment of IPF. This is a tremendous shift for patients, providers and other stakeholders in this disease. In our judgment, there do not appear to be qualitative differences in efficacy between pirfenidone and nintedanib, and it is unclear at this time whether one medication or the other should be used first, whether these medications should be used in combination, and what constitutes treatment failure. There may be differences in labelling that determine choice of drug for some patients, and the different side effect profiles may determine choice of drug for others. In the coming years, personalised medicine may identify pirfenidone and nintedanib 'responders' that derive particular benefit from one or both these medications. For the time being, however, usage will likely be driven by individual patient and provider preference. Combination therapy (ie, the coadministration of pirfenidone and nintedanib) is theoretically appealing given the hypothesis that a large number of fibrotic pathways are active in IPF pathogenesis and the possibility of synergistic effects. However, pharmacokinetic and safety data are required before this should be considered, and a high priority should be placed on testing combination therapy for additive benefit.

Importantly, neither pirfenidone nor nintedanib is a cure for IPF. Neither drug improves lung function and, on average, patients on these therapies continue to progress. Further, we do not know if these drugs remain effective beyond 52 weeks; it is possible that their benefit wanes as time goes on. Therefore, the comprehensive care of patients with IPF remains essential, which includes careful risk prediction, management of comorbidities and physical debility, monitoring for disease progression, and timely referral for lung transplantation. It is also critical that we continue to encourage patients with IPF to participate in clinical trials of new drug agents that will undoubtedly add benefit to these initial therapies.

Ongoing and future trials will need to adapt to the presence of pirfenidone and nintedanib, most likely by allowing their coadministration. In some cases, this will require additional pharmacokinetic and safety data, and will increase the sample size required for adequately powered traditional endpoints. Clinical trials, including more physiologically advanced patients may improve efficiency, as this population appears to have a greater likelihood of clinical events (eg, disease progression, acute exacerbation, death). Importantly, the IPF community needs to streamline the clinical trial process in IPF by working to simplify subject recruitment and standardise key elements of study design, in particular the choice of primary endpoints for efficacy trials.

\section{CONCLUSIONS AND FUTURE DIRECTIONS}

Recent data show that pirfenidone and nintedanib slow progression of mild to moderate IPF, while acetylcysteine has no benefit. We hope that the identification of effective therapies for IPF will provide additional incentive to sponsors to enter the IPF field, and will lead to development of more effective and bettertolerated treatment options. Patients with IPF continue to await a cure for their disease, and the unmet medical need remains high. We believe, however, that the impact of pirfenidone and nintedanib on IPF disease progression represents an important step toward improving these patients' lives.

Competing interests CJR has received fees for serving on advisory boards from InterMune and Boehringer-Ingelheim, lecture fees from InterMune and Boehringer-Ingelheim, and grant support from InterMune. HRC receives grant support from the National Institute of Health, has research contracts with Boehringer Ingelheim, and has consulting contracts with Bayer, Biogen, FibroGen, Genentech, Genoa, Gilead, InterMune, Moerae matrix, Pfizer, Promedior, and Takeda.
Provenance and peer review Not commissioned; internally peer reviewed.

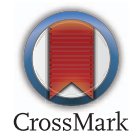

To cite Ryerson CJ, Collard HR. Thorax 2014;69:791792.

Received 12 June 2014

Accepted 13 June 2014

Published Online First 4 July 2014

Thorax 2014;69:791-792.

doi:10.1136/thoraxinl-2014-205892

\section{REFERENCES}

1 Raghu G, Collard HR, Egan JJ, et al. An official ATS/ ERS/JRS/ALAT statement: idiopathic pulmonary fibrosis: evidence-based guidelines for diagnosis and management. Am J Respir Crit Care Med 2011;183:788-824.

2 Ley B, Ryerson CJ, Vittinghoff $\mathrm{E}$, et al. A multidimensional index and staging system for idiopathic pulmonary fibrosis. Ann Intern Med 2012;156:684-91.

3 American Thoracic Society. Idiopathic pulmonary fibrosis: diagnosis and treatment. International consensus statement. American Thoracic Society (ATS), and the European Respiratory Society (ERS). Am J Respir Crit Care Med 2000;161(2 Pt 1): 646-64.

4 Richeldi L, du Bois RM, Raghu G, et al. Efficacy and safety of nintedanib in idiopathic pulmonary fibrosis. N Engl J Med 2014;370:2071-82.

5 King TE Jr, Bradford WZ, Castro-Bernardini S, et al. A phase 3 trial of pirfenidone in patients with idiopathic pulmonary fibrosis. $N$ Engl I Med 2014:370:2083-92.

6 Martinez FJ, de Andrade JA, Anstrom KJ, et al.; Idiopathic Pulmonary Fibrosis Clinical Research Network. Randomized trial of acetylcysteine in idiopathic pulmonary fibrosis. N Engl I Med 2014;370:2093-101.

7 Azuma A, Nukiwa T, Tsuboi E, et al. Double-blind, placebo-controlled trial of pirfenidone in patients with idiopathic pulmonary fibrosis. Am I Respir Crit Care Med 2005;171:1040-7.

8 Taniguchi $\mathrm{H}$, Ebina $\mathrm{M}$, Kondoh $\mathrm{Y}$, et al. Pirfenidone in idiopathic pulmonary fibrosis. Eur Respir J 2010;35:821-9.

9 Raghu G, Johnson WC, Lockhart D, et al. Treatment of idiopathic pulmonary fibrosis with a new antifibrotic agent, pirfenidone: results of a prospective, open-label Phase II study. Am I Respir Crit Care Med 1999:159(4 Pt 1):1061-9.

10 Noble PW, Albera C, Bradford WZ, et al. Pirfenidone in patients with idiopathic pulmonary fibrosis (CAPACITY): two randomised trials. Lancet 2011:377:1760-9.

11 Richeldi L, Costabel U, Selman M, et al. Efficacy of a tyrosine kinase inhibitor in idiopathic pulmonary fibrosis. N Engl J Med 2011:365:1079-87.

12 Demedts M, Behr J, Buhl R, et al. High-dose acetylcysteine in idiopathic pulmonary fibrosis. N Engl J Med 2005;353:2229-42.

13 Raghu G, Anstrom KJ, King TE Jr, et al.; Idiopathic Pulmonary Fibrosis Clinical Research Network. Prednisone, azathioprine, and $\mathrm{N}$-acetylcysteine for pulmonary fibrosis. $N$ Engl I Med 2012;366:1968-77. 